\title{
Diagnosis of Bloodstream Infections by Mass Spectrometry: Present and Future
}

\author{
Walter Florio1, Paola Morici ${ }^{1}$, Cosmeri Rizzato², Simona Barnini ${ }^{3}$, Arianna Tavanti ${ }^{2}$ and Antonella Lupetti ${ }^{1 *}$ \\ ${ }^{1}$ Dipartimento di Ricerca Traslazionale e delle Nuove Tecnologie in Medicina e Chirurgia, Università di Pisa, via San Zeno 37-39, 56127 Pisa, Italy \\ ${ }^{2}$ Dipartimento di Biologia, Università di Pisa, via San Zeno 37-39, 56127 Pisa, Italy \\ ${ }^{3}$ Azienda Ospedaliero-Universitaria Pisana, via Paradisa 2, 56124 Pisa, Italy
}

\begin{abstract}
Rapid identification and antimicrobial susceptibility testing of the causative agent(s) of bloodstream infections may impact on the clinical outcome of patients, which is directly related to the prompt administration of an effective antimicrobial therapy. Empirical antimicrobial therapy is chosen on the basis of clinical and epidemiological data and it is administered immediately after blood sampling but, in a significant number of cases, it has to be streamlined on the basis of the microbiological report. Rapid identification has a clinically relevant impact on the timely selection of an appropriate antimicrobial therapy, especially in low-prevalence areas for antimicrobial resistance. Recently, the identification process of isolated bacteria has been revolutionized by the introduction of mass spectrometry (MS), particularly MALDI-TOF, in clinical microbiology laboratories. Furthermore, MALDI-TOF is one of the most promising techniques for the identification of bacterial and fungal infectious agents directly from positive blood cultures and a potentially useful tool for the detection of antimicrobial resistance, specifically that conferred by $\beta$-lactamases. Although blood culture remains, at present, the gold standard to diagnose bloodstream infections, newly developed MALDI-TOF methods are useful adjunctive tests to fasten the diagnostic process and further increase the diagnostic yield.
\end{abstract}

Keywords: Mass spectrometry; MALDI-TOF; Bacterial identification; Fungal identification; Antimicrobial susceptibility testing; Bloodstream infections

\section{Introduction}

Bloodstream infections (BSI) are a leading cause of death among critically ill patients. Despite the efforts made to improve diagnosis and management, the incidence and number of deaths related to these infections are increasing, often due to inadequate antimicrobial therapy administered within the first $24 \mathrm{~h}$ [1-3]. In addition, empirical antimicrobial therapy may favor the selection and spread of antimicrobial resistant pathogens, and increase the frequency of invasive fungal infections [4]. Rapid identification (ID) and antimicrobial susceptibility testing (AST) of the causative agent(s) of BSI are among the most important tasks of the clinical microbiology laboratory since this information is essential for clinicians to select the most appropriate antimicrobial therapy. Recently, the identification process of isolated bacteria has been revolutionized by the introduction of mass spectrometry (MS), particularly MALDI-TOF, in clinical microbiology laboratories.

The aim of the present work is to review and discuss recent technological developments of the implementation of mass spectrometry to identification of bacterial and fungal infectious agents and possible characterization of their resistance determinants, the understanding of which could provide new perspectives to further improve and shorten the microbiological diagnosis.

\section{Blood culture: The gold standard}

Blood culture is the gold standard for the diagnosis of BSI. Blood cultures are widely used to discriminate whether a febrile episode is due to an infectious or a non-infectious cause and to provide information about the infectious agent, i.e., species ID and susceptibility testing to a panel of antimicrobials. The most relevant advances in blood culture technology have been made during the last decades of the $20^{\text {th }}$ century with the introduction of automated blood culture systems, continuously monitoring the microbial growth. Blood culture is usually performed on at least two separate blood samples taken at different times, with more blood samples resulting in higher detection rates [5]. Blood culture sensitivity may vary significantly depending on the number of blood samples that are taken for each patient and sample volume. Within 5 days of incubation, $99 \%$ of bloodstream infections can be detected using Bactec blood culture bottles [6]. Automated blood culture systems can detect as few as 1 colony forming unit $/ \mathrm{ml}$. The culture time for bacteria to grow to detectable levels by an automated instrument is approximately 12 to 24 hours of incubation for Gram-negative or 24 to 48 hours for Gram-positive microorganisms. Longer incubation times and culture medium additives may be required when fastidious microorganisms are suspected [7]. The current method to diagnose BSI involves an overnight subculture from positive blood cultures and subsequent species ID and AST of isolated microorganisms by an automated system [8]. While the results of species ID and AST of the microorganism by the current method are reported not earlier than 48 $\mathrm{h}$ after blood culture positivity, the Gram staining result is reported by phone to the physician rather quickly (Figure 1). The notification of Gram staining results in less than $1 \mathrm{~h}$, influencing the timely selection of an appropriate antimicrobial therapy, has been associated with a significantly reduced mortality in patients with BSI, compared with delayed processing [9].

\section{Variations to the standard: Direct inoculation methods}

To shorten the turnaround time required for diagnosis of BSI, the fluid from a positive blood culture bottle may be directly inoculated into an automated system for ID and AST of bacteria, further referred to as direct methods (Figure 1). Several studies have compared the results of the current blood culture method with those by direct methods [10-18]. The direct method has been shown to yield reliable results

*Corresponding author: Antonella Lupetti, Dipartimento di Ricerca Traslazionale e delle Nuove Tecnologie in Medicina e Chirurgia, Università di Pisa, Via San Zeno 37-39, 56127 Pisa, Italy, Tel: +39-050-2213722; E-mail: antonella.lupetti@med.unipi.it

Received September 29, 2015; Accepted October 04, 2015; Published October 11,2015

Citation: Florio W, Morici P, Rizzato C, Barnini S, Tavanti A, et al. (2015) Diagnosis of Bloodstream Infections by Mass Spectrometry: Present and Future. Mass Spectrom Open Access 1: 106. doi:10.4172/mso.1000106

Copyright: $\odot 2015$ Florio W, et al. This is an open-access article distributed under the terms of the Creative Commons Attribution License, which permits unrestricted use, distribution, and reproduction in any medium, provided the original author and source are credited. 


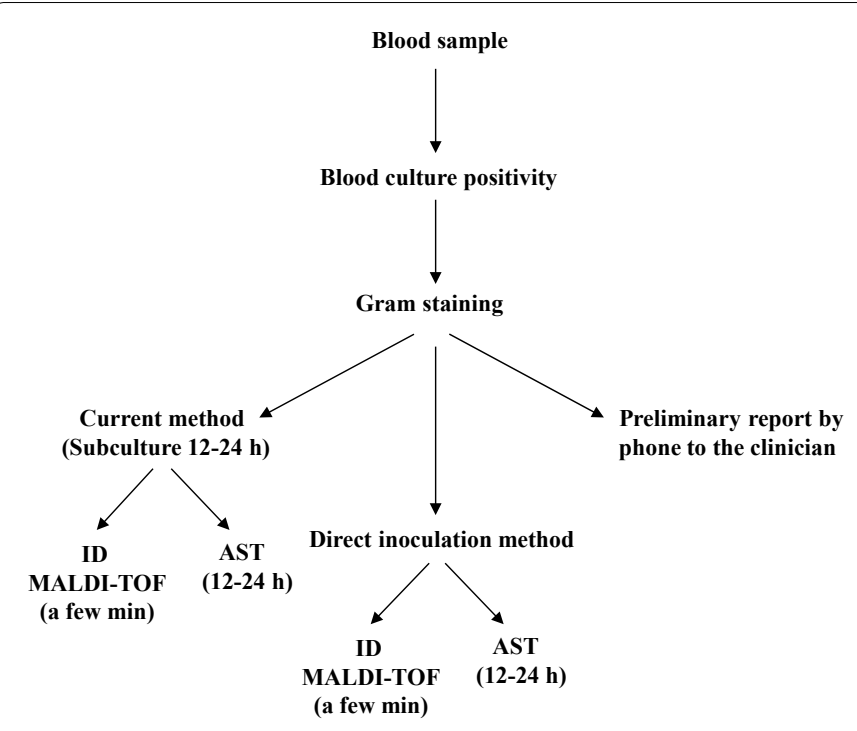

Figure 1: Schematic representation of the various steps of the different diagnostic procedures described in the text.

for Gram-negative rods (being better for Enterobacteriaceae than for Pseudomonas aeruginosa) and, recently, also for Gram-positive cocci $[14,15,17,18]$. Treatment with detergents, such as saponin, before inoculation into the appropriate ID and AST cards improves the recovery of Gram-positive cocci as well as $P$. aeruginosa from positive blood culture, probably by releasing intracellular bacteria from human blood cells [18]. Although some isolates may be missed or misidentified by the direct method, a good categorical agreement between the direct and standard methods is usually observed for AST results, with relatively low overall error rates for the direct method $[13,14,17,18]$. The main sources of errors by direct methods are mixed cultures and a too small inoculum size. As a small (6-10\%), but significant, percentage of specimens appearing monomicrobial at the Gram staining results to be polymicrobial after subculture $[11,13,14,19]$ the results obtained with the direct method should be considered preliminary until the inoculum has been confirmed to be monomicrobial by subculture. This does not delay the report of results as the mono/polymicrobial nature of specimens can be assessed on subcultures inoculated the previous day. As an alternative to the direct methods, the AST can be accomplished after a short-term subculture $(<6 \mathrm{~h})$ in solid medium [20] with more reliable results for Gram-negative (97.6\%) than for Gram-positive (64\%) bacteria [20].

Rapid methods for AST represent an important advancement in the diagnosis of BSI as the results are available 12-24 h earlier than with the current method, depending on the chosen method and on how the workflow is organized, thus allowing a rapid selection of the appropriate antimicrobial therapy.

\section{MALDI-TOF MS identification of bacteria causing bloodstream infections}

Mass spectrometry (MS) is a highly accurate analytical technique that allows measuring the mass/charge ratio of a variety of compounds. Matrix-assisted laser desorption/ionization time of flight (MALDITOF) is the most widely used MS technique for the analysis of biomolecules, particularly for polypeptides. In recent years, MALDITOF MS has become an effective instrument for identification of bacteria from isolated colonies. For microbial identification, the molecular mass range analyzed is between 2 and $20 \mathrm{kDa}$, which is very stable and with a strong signal to noise ratio. This size range is dominated by ribosomal proteins, which provide accurate spectra, and are not markedly influenced by microbial growth conditions. Identification is obtained by matching the most similar spectrum in the database to that of the unknown microorganism. The software that compares the spectra generates a numerical value (score value) based on the similarities between the observed and stored data sets. In general, a score value above 2.0 is considered a valid species identification, whereas values between 2.0 and 1.7 represent reliable genus identification, though it has been proposed that cut-off values could be lowered down to 1.4 without compromising the accuracy of results [21-25].

\section{Rapid MALDI-TOF MS identification of bacteria causing bloodstream infections}

MALDI-TOF MS can identify bacteria within a few minutes and only a small amount of microbial biomass is required for analysis. For these reasons, MALDI-TOF MS is one of the most promising techniques for the identification of infectious agents directly from positive blood culture [23,26-30].

Better performances have been described for Gram-negative than for Gram-positive bacteria [22,25,31,32]. Differences in the sample processing methods may account, at least in part, for the observed variability in correct identification rates [33]. In order to minimize possible interferences by other proteins, several sample preparation protocols have been developed that separate bacterial and host cells and proteins through cell lysis and/or differential centrifugation steps [34-36]. These protocols usually yield bacterial samples largely devoid of blood components but may be laborious and not very effective in recovering microorganisms from $\mathrm{BC}$ containing low bacterial loads. In fact, it has been shown by spike/dilution experiments that the number of bacteria in $\mathrm{BC}$ may be a critical factor for successful identification by MALDI-TOF [36]. Moreover, due to the additional processing time, most microbiological laboratories use these methods in batches for organizational reasons, thus reducing the gain in time obtained by a faster method. In addition, the significant hands on time of the various sample preparation protocols described in the literature may hinder their implementation in laboratories with limited human resources. As an alternative to differential centrifugations, bacteria can be separated from BC using serum separator tubes (SST), which is rapid and allows to purify bacteria with reasonably good yields. Sporadically, removal of blood cells by SST centrifugation may be not very efficient and, therefore, a few samples should be processed a second time.

The final step in sample preparation for MALDI-TOF analysis is lysis of bacteria and extraction of intracellular proteins. This may be effectively achieved by a standard ethanol/formic acid protein extraction protocol, followed by centrifugation to remove intact bacteria and bacterial cell debris [34-36]. Alternatively, bacterial lysis and extraction of bacterial proteins may be carried out directly on the steel target plate before addition of matrix solution [25,37]. To this regard, a method which is rapid, reliable, simple, and easy to integrate into the diagnostic routine has been proposed recently by Barnini et al. [25] in order to be fully applicable to diagnostic routine, without the need to work in batches.

\section{Assessment of $\beta$-lactamase mediated resistance by MALDI- TOF}

MALDI-TOF MS is a potentially useful tool for the detection of antimicrobial resistance, particularly that conferred by $\beta$ -lactamases [38-41]. Resistance to $\beta$-lactam antibiotics can be tested 
by MS as hydrolysis of the $\beta$-lactam ring by $\beta$-lactamases results in the disappearance of the original mass peak of the antibiotic and appearance of the corresponding products generated by hydrolysis [42]. On the contrary, mechanisms of resistance other than those mediated by $\beta$-lactamases, such as mutated porins and up-regulation of efflux pumps, cannot be detected by MALDI-TOF, at present.

A few studies have been performed to discriminate methicillinresistant (MRSA) from susceptible (MSSA) Staphylococcus aureus strains using MALDI-TOF MS. Considerable diversity between MALDITOF fingerprints from different MRSA strains has been demonstrated, suggesting the general feasibility of MALDI-TOF-based discrimination of MRSA lineages. Of course, further independent clonal complexes should be analyzed and incorporated into the typing scheme to become a rapid first-line screening tool for prediction of clonal lineages and outbreak investigation. Employing routine real-time typing has the potential to improve MRSA surveillance [43]. Discordant results are often due to systematic database-related taxonomical differences that can be reduced in the future with an expanding database.

Another, recently described application of MALDI-TOF MS for the detection of antimicrobial resistance is based on the identification of $b l a_{K P C}$ carbapenemase gene-bearing plasmids conferring carbapenem resistance. Rapid methods for tracking plasmids carrying carbapenemase genes could greatly benefit infection control efforts [44]. Plasmid identification by MALDI-TOF MS can be accomplished in $10 \mathrm{~min}$ from isolated colonies and $30 \mathrm{~min}$ from positive blood cultures, thus showing the potential clinical utility of this method.

All together, MALDI-TOF MS allows rapid identification of bacteria $[45,46]$ and potentially provides important information about $\beta$-lactamase-mediated resistance, which may support the early start of an appropriate antimicrobial therapy.

\section{Rapid MALDI-TOF MS identification of fungi causing bloodstream infections}

Fungal systemic infections are on the rise and their increasing incidence has been linked to recent advanced treatment strategies for long degenerative pathologies, which have led to a higher number of immunocompromised patients [47]. Systemic infections due to Candida spp. are characterized by a high mortality rate, especially when associated to septic shock, with percentages rising to up to $97.6 \%$ if patients are not treated with adequate antifungal therapy within 24 hours [48]. The possibility of a rapid identification of fungal species directly from blood samples would significantly speed up routine workflow and a reduction in turnaround time, ultimately benefitting the patient. To this end, MALDI-TOF MS has been proven to provide an early diagnosis of yeast species directly from blood cultures, even though the different protocols used may yield variable outcomes [22,48-52]. A recent paper by Idelevich et al. investigated the usefulness of a combined approach in which MALDI-TOF MS identification is applied to very short-term blood cultures [53]. The same biomass from very short-term blood cultures is also used for an early inoculation of an automated susceptibility testing device [53]. The ability of mass spectrometry to identify different yeast species was confirmed when this technique was directly applied to early positive blood cultures, even though the success rate was strongly associated with the fungal concentration in the sample, as also reported by others [51]. However, when yeast cell pellets prepared with the same standard kit protocol were used for antifungal susceptibility testing with the main aim to provide an early response on drug susceptibility, performance was sub optimal [53]. In fact, for some of the isolates the test aborted, most likely because of the limited number of fungal cells in the starting inoculum. This result clearly indicates that conventional testing from subcultured isolates is still necessary for validation of the results [53].

More recently, an innovative MALDI-TOF MS-based approach to identify a specific signature of bloodstream infections caused by Candida albicans was described by Sendid et al. [54]. A similar approach was previously used in the set up with tests aimed at detecting Candida cell wall moieties (e.g., mannans, glucans) by immunocapture or biochemical techniques in sera of patients with candidaemia [55]. These tests are particularly interesting, as one of the major issues in the management of systemic infections is the difficulty of an early diagnosis, which is required for a favourable outcome. Following optimization of pre-analytical procedures, Sendid et al. [54] aimed at extracting oligosaccharides from serum and were able to identify a specific peak of $\mathrm{m} / \mathrm{z} 365$ by mass spectrum analysis, which was identified as a disaccharide. Interestingly, this disaccharide was specifically associated to sera obtained from patients with candidaemia and it was absent in control sera collected from healthy subjects. Mouse models of Candida albicans colonization and infection showed that this signal was specifically associated with tissue invasion, suggesting that clinical evaluation of its usefulness in discriminating colonized and infected patients would be feasible. Such a study highlights the potentiality of this mass spectrometry-based method to rapidly discriminate between colonized individuals and patients with disseminated candidiasis based on the presence of this biomarker [54].

\section{Combining PCR with MS for rapid diagnosis of BSI}

Another promising application of mass spectrometry to the identification of microorganisms causing bloodstream infections is ElectroSpray Ionization Mass Spectrometry (ESI-MS) used on the amplicons generated by broad-spectrum PCR to potentially identify any microorganism present in a clinical specimen [56]. Using mass spectrometry, the mass of each PCR amplicon is determined and the nucleotide base composition is calculated and compared to a database, achieving the identification of the pathogen. Microbial identification in positive blood culture aliquots by PCR/ESI-MS showed $96.8 \%$ sensitivity and $98.5 \%$ specificity in comparison with the current method [57]. When PCR/ESI-MS was performed in whole blood specimens, thus avoiding culture, sensitivity and specificity for the molecular method were $50 \%$ and $93.8 \%$, respectively. The accuracy of the PCR/ESI-MS method may be comparable to that of a real-time PCR-based system [57], though the two methods may have advantages and drawbacks. Real-time PCR is very rapid and relatively simple to perform but the number of detectable microbial species is limited to predefined panels of 25-60 species. The PCR/ESI-MS method can identify more than 400 species of bacteria with a reasonable turnaround time (6-8 $\mathrm{h}$ ) and has a high-throughput capacity but it is more technically complex than realtime PCR, and it may be more difficult to integrate into the standard laboratory workflow.

Specific PCR-based assays, such as the Xpert MRSA/SA assay, have been developed and evaluated for their ability to identify in $1 \mathrm{~h} S$. aureus and methicillin-resistance from blood cultures with Gram-positive cocci in clusters and tetrads [58,59]. The Xpert MRSA/SA BC (Cepheid, Sunnyvale, CA) is a real-time PCR-based method that detects sequences within the staphylococcal protein A ( $s p a)$ gene, the gene for methicillin resistance ( $m e c A$ ), and the staphylococcal cassette chromosome (SCCmec) inserted into the $S$. aureus chromosomal attB insertion site. Inclusion of the attB insertion site and the mecA gene targets enables the assay to discriminate methicillin-resistant $S$. aureus from methicillin-resistant coagulasenegative Staphylococcus species that may occur in molecular tests that target only the SCCmec cassette. In a recent report, Romero-Gómez et al. 
[59] described that the sensitivity and specificity of the Xpert MRSA/SA BC assay were $96.0 \%$ and $100 \%$, respectively. The accuracy of the method combining the MALDI-TOF and Xpert MRSA/SA BC systems was $98.9 \%$, with a negative and positive predictive value of $98.5 \%$ and $100 \%$, respectively [59]. However, one limitation of the Xpert is the presence of mutations or polymorphisms in primer and probe binding regions, which may affect detection of new or unknown MRSA variants, resulting in a false-negative result. The PCR/MS technologies could fasten and increase the detection of microbial pathogens in BSI but, at present, they cannot replace conventional microbiological methods for the assessment of antimicrobial susceptibility of the etiologic agent, and still require further substantial investigation before their inclusion in routine use as adjunctive tests.

\section{Conclusions}

An ideal method for the diagnosis of BSI should rapidly identify the infectious agent(s) and its antimicrobial susceptibility profile in order to timely select an effective antimicrobial therapy. At present, an empirical antimicrobial therapy is chosen on the basis of clinical and epidemiological data and it is administered immediately after blood sampling. Rapid ID has a clinically relevant impact to streamline the appropriate antimicrobial therapy. To this regard, at present the MALDI-TOF based direct method represents the best option for microbial ID, as it is rapid, reliable, and relatively inexpensive. Moreover, since the impact of rapid ID on antimicrobial selection varies significantly between low- and high-prevalence areas for antibiotic resistance [29], we believe that a rapid method for AST should also be performed. To this aim, at present, the previously described direct inoculation methods are a valid option to provide accurate results 12$24 \mathrm{~h}$ earlier than the current method. The recently described results for the detection of bacterial $\beta$-lactamase resistance by MALDI-TOF are interesting and promising, although further research is required to establish effective methodologies allowing wider detection of resistance determinants by mass spectrometry.

Another important issue regards the additional processing time required by some of these new methods, which may hinder their implementation in clinical microbiology laboratories. Additional studies focused on simplification of diagnostic procedures will facilitate the complete integration of newly developed methods into the diagnostic routine.

\section{Acknowledgments}

The Authors pay tribute to the late Prof. Mario Campa for his long standing, inspiration and support. This study was supported by a research grant from the Italian "Ministero dell'Istruzione, dell'Università e della Ricerca", contract $n^{\circ}$ 2012WJSX8K_005.

\section{Declaration of interest}

The authors report no conflicts of interest. The authors alone are responsible for the content and writing of this article.

\section{References}

1. Kumar A, Ellis P, Arabi Y, Roberts D, Light B, et al. (2009) Initiation of inappropriate antimicrobial therapy results in a fivefold reduction of survival in human septic shock. Chest 136: 1237-1248.

2. Carlet J, Collignon P, Goldmann D, Goossens H, Gyssens IC , et al. (2011) Society's failure to protect a precious resource: antibiotics. Lancet 378: 369-371.

3. Lagu T, Rothberg MB, Shieh MS, Pekow PS, Steingrub JS, et al. (2012) What is the best method for estimating the burden of severe sepsis in the United States? J Crit Care 27: 414.e1-9.

4. Charles PE, Dalle F, Aube H, Doise JM, Quenot JP, et al. (2005) Candida spp. colonization significance in critically ill medical patients: a prospective study. Intensive Care Med 31: 393-400.
5. Lee A, Mirrett S, Reller LB, Weinstein MP (2007) Detection of bloodstream infections in adults: how many blood cultures are needed? J Clin Microbiol 45 3546-3548.

6. Cockerill FR 3rd, Wilson JW, Vetter EA, Goodman KM, Torgerson CA, et al. (2004) Optimal testing parameters for blood cultures. Clin Infect Dis 38: 1724-1730.

7. Kirn TJ, Weinstein MP (2013) Update on blood cultures: how to obtain, process, report, and interpret. Clin Microbiol Infect 19: 513-520.

8. Jordan JA, Durso MB (2005) Real-time polymerase chain reaction for detecting bacterial DNA directly from blood of neonates being evaluated for sepsis. J Mol Diagn; 7: 575-581.

9. Barenfanger J, Graham DR, Kolluri L, Sangwan G, Lawhorn J, et al. (2008) Decreased mortality associated with prompt Gram staining of blood cultures. Am J Clin Pathol 130: 870-876.

10. Bruins MJ, Bloenberger P, Ruijs GJ, Wolfhagen MJ (2004) Identification and susceptibility testing of Enterobacteriaceae and Pseudomonas aeruginosa by direct inoculation from positive Bactec blood culture bottles into Vitek 2. J Clin Microbiol 42: 7-11.

11. de Cueto M, Ceballos E, Martinez-Martinez L, Perea EJ, Pascual A (2004) Use of positive blood cultures for direct identification and susceptibility testing with the Vitek 2 system. J Clin Microbiol 42: 3734-3738.

12. Funke G, Funke-Kissling P (2004) Use of the BD Phoenix automated microbiology system for direct identification and susceptibility testing of Gramnegative rods from positive blood cultures in a three-phase trial. J Clin Microbiol 42: $1466-1470$.

13. Lupetti A, Barnini S, Castagna B, Capria AL, Nibbering PH (2010) Rapid identification and antimicrobial susceptibility profiling of Gram-positive cocci in blood cultures with the Vitek 2 system. Eur J Clin Microbiol Infect Dis 29: 89-95.

14. Lupetti A, Barnini S, Castagna B, Nibbering PH, Campa M (2010) Rapid identification and antimicrobial susceptibility testing of Gram-positive cocci in blood cultures by direct inoculation into the BD Phoenix system. Clin Microbiol Infect 16: 986-991

15. Gherardi G, Angeletti S, Panitti M, Pompilio A, Di Bonaventura G, et al. (2012) Comparative evaluation of the Vitek-2 Compact and Phoenix systems for rapid identification and antibiotic susceptibility testing directly from blood cultures of Gram-negative and Gram-positive isolates. Diagn Microbiol Infect Dis 72: 20-31.

16. Yonetani S, Okazaki M, Araki, K, Makino H, Fukugawa Y, et al. (2012) Direct inoculation method using BacT/Alert 3D and BD Phoenix system allows rapid and accurate identification and susceptibility testing for both Gram-positive cocci and Gram-negative rods in aerobic blood cultures. Diagn Microbiol Infect Dis 73: $129-134$.

17. Beuving J, van der Donk CF, Linssen CF, Wolffs PF, Verbon A (2011) Evaluation of direct inoculation of the BD Phoenix system from positive Bactec blood cultures for both Gram-positive cocci and Gram-negative rods. BMC Microbiol 11: 156.

18. Lupetti A, Barnini S, Morici P, Ghelardi E, Nibbering PH, et al. (2013) Saponin promotes rapid identification and antimicrobial susceptibility profiling of Grampositive and Gram-negative bacteria in blood cultures with the Vitek 2 system. Eur J Clin Microbiol Infect Dis 32: 493-502.

19. Waites KB, Brookings ES, Moser SA, Zimmer BL (1998) Direct bacteria identification from positive BacT/Alert blood cultures using MicroScan overnight and rapid panels. Diagn Microbiol Infect Dis 32: 21-26.

20. Idelevich EA, Schüle I, Grünastel B, Wüllenweber J, Peters G, et al. (2014) Rapid identification of microorganisms from positive blood cultures by MALDITOF mass spectrometry subsequent to very short-term incubation on solid medium. Clin Microbiol Infect 20: 1001-1006

21. Seng P, Drancourt M, Gouriet F, La Scola B, Fournier PE, et al. (2009) Ongoing revolution in bacteriology: routine identification of bacteria by matrix-assisted laser desorption ionization time-of-flight mass spectrometry. Clin Infect Dis 49 543-551.

22. Buchan BW, Riebe KM, Ledeboer NA (2012) Comparison of the MALDI Biotype system using Sepsityper specimen processing to routine microbiological methods for identification of bacteria from positive blood culture bottles. J Clin Microbiol 50: 346-352.

23. Lagacé-Wiens PR, Adam HJ, Karlowsky JA, Nichol KA, Pang PF, et al. (2012) Identification of blood culture isolates directly from positive blood cultures by use of matrix-assisted laser desorption ionization-time of flight mass spectrometry and a commercial extraction system: analysis of performance, cost, and turnaround time. J Clin Microbiol 50: 3324-3328. 
24. Saffert RT, Cunningham SA, Mandrekar J, Patel R (2012) Comparison of three preparatory methods for detection of bacteremia by MALDI-TOF mass spectrometry. Diagn Microbiol Infect Dis 73: 21-26

25. Barnini S, Ghelardi E, Brucculeri V, Morici P, Lupetti A (2015) Rapid and reliable identification of Gram-negative bacteria and Gram-positive cocci by deposition of bacteria harvested from blood cultures onto the MALDI-TOF plate. BMC Microbiol 15: 124

26. La Scola B, Raoult D (2009) Direct identification of bacteria in positive blood culture bottles by matrix-assited laser desorption ionization time-of-flight mass spectrometry. PLoS One 4: e8041.

27. Cherkaoui A, Hibbs J, Emonet S, Tangomo M, Girard M, et al. (2010) Comparison of two matrix-assisted laser desorption ionization-time of fligh mass spectrometry methods with conventional phenotypic identification for routine identification of bacteria to the species level. J Clin Microbiol 48: 11691175 .

28. Jamal W, Saleem R, Rotimi VO (2013) Rapid identification of pathogens directly from blood culture bottles by Bruker matrix-assisted laser desorption laser ionization-time of flight mass spectrometry versus routine methods. Diagn Microbiol Infect Dis 76: 404-408.

29. Clerc O, Prod'hom G, Vogne C, Bizzini A, Calandra T, et al. (2013) Impact of matrix-assisted laser desorption ionization time-of-flight mass spectrometry on the clinical management of patients with Gram-negative bacteremia: a prospective observational study. Clin Infect Dis 56: 1101-1107.

30. Machen A, Drake T, Wang YF (2014) Same day identification and full pane antimicrobial susceptibility testing of bacteria from positive blood culture bottles made possible by a combined lysis-filtration method with MALDI-TOF Vitek mass spectrometry and the Vitek 2 system. PLoS One 9: e87870.

31. Moussaoui W, Jaulhac B, Hoffmann AM, Ludes B, Kostrzewa M, et al. (2010) Matrix-assisted laser desorption ionization time-of-flight mass spectrometry identifies $90 \%$ of bacteria directly from blood culture vials. Clin Microbiol Infect 16: $1631-1638$.

32. Kok J, Thomas LC, OIma T, Chen SC, Iredell JR (2011) Identification of bacteria in blood culture broths using matrix-assisted laser desorption-ionization Sepsityper and time of flight mass spectrometry. PLoS One 6: e23285

33. Loonen AJ, Jansz AR, Stalpers J, Wolffs PF, van den Brule AJ (2012) An evaluation of three processing methods and the effect of reduced culture times for faster direct identification of pathogens from BacT/Alert blood cultures by MALDI-TOF MS. Eur J Clin Microbiol Infect Dis 31: 1575-1583.

34. Christner M, Rohde H, Wolters M, Sobottka I, Wegscheider K, et al. (2010) Rapid identification of bacteria from positive blood culture bottles by use of matrix-assisted laser desorption ionization time of flight mass spectrometry fingerprinting. J Clin Microbiol 48: 1584-1591.

35. Stevenson LG, Drake SK, Murray PR (2010) Rapid identification of bacteria in positive blood culture broths by matrix-assisted laser desorption ionization-time of flight mass spectrometry. J Clin Microbiol 48: 444-447.

36. Schubert S, Weinert K, Wagner C, Gunzl B, Wieser A, et al. (2011) Novel, improved sample preparation for rapid, direct identification from positive blood cultures using matrix-assisted laser desorption/ionization time-of-flight (MALDITOF) mass spectrometry. J Mol Diagn 13: 701-706

37. Ferroni A, Suarez S, Beretti J-L, Dauphin B, Bille E, et al. (2010) Real-time identification of bacteria and Candida species in positive blood culture broths by matrix-assisted laser desorption ionization-time of flight mass spectrometry. J Clin Microbiol 48: 1542-1548.

38. Burckhardt I, Zimmermann S (2011) Using matrix-assisted laser desorption ionization-time of flight mass spectrometry to detect carbapenem resistance within 1 to 2.5 hours. J Clin Microbiol 49: 3321-3324.

39. Hooff GP, van Kampen JJ, Meesters RJ, van Belkum A, Goessens WH, et al. (2012) Characterization of $\beta$-lactamase enzyme activity in bacterial lysates using MALDI-mass spectrometry. J Proteome Res 11: 79-84.

40. Hrabák J, Studentová V, Walková R, Zemlicková H, Jakubu V, et al. (2012) Detection of NDM-1, VIM-1, KPC, OXA-48, and OXA-162 carbapenemases by matrix-assisted laser desorption ionization-time of flight mass spectrometry. $J$ Clin Microbiol 50: 2441-2443.

41. Sparbier K, Schubert S, Weller U, Boogen C, Kostrzewa M (2012) Matrixassisted laser desorption ionization-time of flight mass spectrometry-based functional assay for the rapid detection of resistance against $\beta$-lactam antibiotics. J Clin Microbiol 50: 927-937.
42. Jung JS, Popp C, Sparbier K, Lange C, Kostrzewa M, et al. (2014) Evaluation of matrix-assisted laser desorption ionization-time of flight mass spectrometry for rapid detection of $\beta$-lactam resistance in Enterobacteriaceae derived from blood cultures. J Clin Microbiol 52: 924-930.

43. Wolters M, Rohde H, Maier T, Belmar-Campos C, Franke G, et al. (2011) MALDI-TOF MS fingerprinting allows for discrimination of major methicillinresistant Staphylococcus aureus lineages. Int J Med Microbiol 301: 64-68.

44. Lau AF, Wang H, Weingarten RA, Drake SK, Suffredini AF, et al. (2014) A rapid matrix-assisted laser desorption ionization-time of flight mass spectrometrybased method for single-plasmid tracking in an outbreak of carbapenemresistant Enterobacteriaceae. J Clin Microbiol 52: 2804-2812.

45. Sauer S, Kliem M (2010) Mass spectrometry tools for the classification and identification of bacteria. Nat Rev Microbiol 8: 74-82.

46. Seng P, Rolain JM, Fournier PE, La Scola B, Drancourt M, et al. (2010) MALDITOF-mass spectrometry applications in clinical microbiology. Future Microbiol 5: 1733-1754.

47. Tortorano AM, Peman J, Bernhardt H, Klingspor L, Kibbler CC, et al. (2004) Epidemiology of candidaemia in Europe: results of 28-month European Confederation of Medical Mycology (ECMM) hospital-based surveillance study. Eur J Clin Microbiol Infect Dis 23: 317-322.

48. Kollef M, Micek S, Hampton N, Doherty JA, Kumar A (2012) Septic shock attributed to Candida infection: importance of empiric therapy and source control. Clin Infect Dis 54: 1739-1746.

49. Ferreira L, Sanchez-Juanes F, Porras-Guerra I, Garcia-Garcia MI, GarciaSanchez JE, et al. (2011) Microorganisms direct identification from blood culture by matrix-assisted laser desorption/ionization time-of-flight mass spectrometry. Clin Microbiol Infect 17: 546-551.

50. Nonnemann B, Tvede M, Bjarnsholt T (2013) Identification of pathogenic microorganisms directly from positive blood vials by matrix-assisted lase desorption/ionization time of flight mass spectrometry. APMIS 121: 871-877.

51. Spanu T, Posteraro B, Fiori B, D'Inzeo T, Campoli S, et al. (2012) Direct MALDITOF mass spectrometry assay of blood culture broths for rapid identification of Candida species causing bloodstream infections: an observational study in two large microbiology laboratories. J Clin Microbiol 50: 176-179.

52. Yan Y, He Y, Maier T, Quinn C, Shi G, et al. (2011) Improved identification of yeast species directly from positive blood culture media by combining Sepsityper specimen processing and Microflex analysis with the matrix-assisted laser desorption ionization Biotyper system. J Clin Microbiol 49: 2528-2532.

53. Idelevich EA, Grunewald CM, Wullenweber J, Becker K (2014) Rapid identification and susceptibility testing of Candida spp. from positive blood cultures by combination of direct MALDI-TOF mass spectrometry and direct inoculation of Vitek 2. PloS one 9: e114834.

54. Sendid B, Poissy J, Francois N, Mery A, Courtecuisse S et al. (2015) Preliminary evidence for a serum disaccharide signature of invasive Candida albicans infection detected by MALDI Mass Spectrometry. Clin Microbiol Infect 21: 88 . e1-6

55. Marchetti O, Lamoth F, Mikulska M, Viscoli C, Verweij P, et al. (2012) European Conference on Infections in Leukemia Laboratory Working, G. ECIL recommendations for the use of biological markers for the diagnosis of invasive fungal diseases in leukemic patients and hematopoietic SCT recipients. Bone Marrow Transplant 47: 846-854

56. Jordana-Lluch E, Carolan HE, Giménez M, Sampath R, Ecker DJ, et al. (2013) Rapid diagnosis of bloodstream infections with PCR followed by mass spectrometry. PLoS One 8: e62108.

57. Jeng K, Gaydos CA, Blyn LB, Yang S, Won H, et al. (2012) Comparative analysis of two broad-range PCR assays for pathogen detection in positiveblood-culture bottles: PCR-high-resolution melting analysis versus PCR-mass spectrometry. J Clin Microbiol 50: 3287-3292.

58. Wolk DM, Struelens MJ, Pancholi P, Davis T, Della-Latta P, et al. (2009) Rapid detection of Staphylococcus aureus and methicillin-resistant S. aureus (MRSA) in wound specimens and blood cultures: multicenter preclinical evaluation of the Cepheid Xpert MRSA/SA skin and soft tissue and blood culture assays. $J$ Clin Microbiol 47: 823-826.

59. Romero-Gómez M-P, Muñoz-Velez M, Gómez-Gil R, Mingorance J (2013) Evaluation of combined use of MALDI-TOF and Xpert MRSA/SA BC assay for the direct detection of methicillin resistance in Staphylococcus aureus from positive blood culture bottles. J Infect 67: 91-92. 